\title{
Dapsone syndrome in a Filipino man
}

\author{
MA. AURORA R. HORTALEZA, NOEMIE \\ G. SALTA-RAMOS, JESUSA BARCELONA-TAN \& \\ MA. LUISA ABAD-VENIDA \\ Department of Dermatology, Jose R. Reyes Memorial Medical \\ Center, Rizal Avenue, Sta. Cruz, Manila, Philippines
}

Accepted for publication 7 March 1995

\begin{abstract}
Summary A case of dapsone syndrome occurring in a Filipino man under treatment for multibacillary (MB) leprosy is described. The patient manifested progressive fever, erythroderma and jaundice 4 weeks after initiation of multidrug therapy (MDT) with rifampicin, clofazimine and dapsone. The clinical symptoms conformed well to the dapsone syndrome first described in the 1950s and this report proves that the syndrome does still exist. There was recovery after dapsone was omitted and therapy with systemic corticosteroids was started.

In view of this potentially fatal hypersensitivity reaction, this case report emphasizes the need for caution when initiating MDT or dapsone therapy. It is also suggested that any patient on MDT or dapsone needs to be referred immediately to a dermatologist or internist if the patient develops a skin rash during the first 2 months of treatment.
\end{abstract}

\section{Introduction}

Dapsone or diaminodiphenylsulphone has been used for over 50 years in the treatment of a variety of dermatologic diseases but its prolonged and extensive use has been in the treatment of leprosy. While a variety of adverse and toxic effects are associated with dapsone, a distinct hypersensitivity reaction has been described. ${ }^{1,2}$ This reaction, termed 'dapsone syndrome' is characterized by the sudden onset of a papular or exfoliative rash, accompanied by fever, malaise and weakness, and followed by jaundice, enlargement and tenderness of the liver, lymphadenopathy and mononucleosis after 2 to 8 weeks of dapsone therapy. However, not all symptoms are necessarily present. ${ }^{1}$ The cutaneous manifestations of the syndrome show wide variations, including erythroderma, papular erythematous eruptions, erythema multiforme, toxic epidermal necrolysis and Stevens Johnson syndrome. ${ }^{3}$

Hypersensitivity reactions to dapsone which were common in the late 1940s and early 
$50 \mathrm{~s}$, then virtually disappeared, were noted to become more frequent in the 1980s. Based on a postal survey by $\mathrm{Smith}^{4}$ (1988), it was not possible to give an overall estimate of the frequency of the dapsone syndrome but it varied from 0 to $2 \%$ of new cases treated.

The fact that few additional cases have been reported since then, despite the millions of leprosy sufferers receiving the drug, is probably related to the lower dosages used and justifies the conclusion that the syndrome is a rare complication of dapsone therapy. ${ }^{5}$

In the Philippines, despite the widespread therapeutic application of dapsone, no case of dapsone syndrome has been reported in the literature. Although this could be a reflection of a true absence of such occurrence, the possible inability of the physicians to recognize the syndrome due to lack of awareness of its existence cannot be totally discounted. We report on the occurrence of this syndrome in a Filipino man who was given the WHO-MDT for MB leprosy.

\section{Case report}

An 18-year-old Filipino male who was seen at the dermatology department of the Jose R. Reyes Memorial Medical Center was diagnosed as having borderline lepromatous leprosy based on anaesthetic skin lesions, a bacteriologic index of $3+$ and skin biopsy findings. He was one week into the WHO-MDT for MB leprosy consisting of rif ampicin ( $600 \mathrm{mg}$ once a month), clofazimine ( $300 \mathrm{mg}$ once a month and $50 \mathrm{mg} /$ day) and dapsone $(100 \mathrm{mg} /$ day $)$ when he developed jaundice. Discontinuation of rifampicin and clofazimine was advised. However, the patient was maintained on dapsone.

Three weeks later, the patient developed fever, and maculopapular rashes on the face and chest. This was confirmed at a provincial hospital where laboratory studies revealed a normal full blood count but elevated liver function tests: SGPT, 874.0 IU/L (normal, 0-31 IU/L), direct bilirubin, $143 \mu \mathrm{mol} / 1$ (normal, $0-3.4 \mu \mathrm{mol} / \mathrm{l}$ ), indirect bilirubin, $182 \mu \mathrm{mol} / 1$ (normal, 3·4-17·0 $\mu \mathrm{mol} / \mathrm{l}$ ) and alkaline phosphatase, $229 \mathrm{IU} / \mathrm{L}$ (normal, 9$45 \mathrm{IU} / \mathrm{L})$. Liver ultrasonography showed diffuse parenchymal disease.

On the ensuing days, he developed mucopurulent eye discharge and blisters on the buccal mucosa, trunk and extremities. The maculopapular rash progressed into generalized scaling. At another hospital, a repeat haematologic study revealed anaemia: haemoglobin of $74 \mathrm{~g} / \mathrm{l}$, erythrocyte count of $2.54 \times 10^{12} / \mathrm{L}$, haematocrit of $25 \%$.

The progression of the patient's condition prompted consultation and eventual admission to the medical centre.

On physical examination, the patient was acutely ill, jaundiced and febrile with a temperature of $38.8^{\circ} \mathrm{C}$. In addition, he had mucopurulent eye discharge and erosions on the buccal mucosa and lips. He had generalized erythema and scaling with multiple tense and flaccid bullae located on the forearms, trunk and feet (Figures 1-4). Erosions and crusted lesions were likewise noted on the trunk and extremities. The liver was palpable but nontender. There was no lymphadenopathy. The rest of the examination was unremarkable.

A complete blood count revealed a haemoglobin of $8.0 \mathrm{~g} / \mathrm{l}$, a haematocrit of $25 \%$, leukocyte count of $21.45 \times 10^{9} / \mathrm{L}$ with lymphocytes of $48 \%$ and platelet count of $150 \times 10^{3} / \mu \mathrm{L}$. Liver function tests were elevated at the following values: indirect bilirubin of $1033.6 \mu \mathrm{mol} / 1$, direct bilirubin of $734.4 \mu \mathrm{mol} / 1$, total bilirubin of $1768 \mu \mathrm{mol} / \mathrm{l}$, alkaline phosphatase of $241 \cdot 4 \mathrm{IU} / \mathrm{L}$ SGOT of $126 \cdot 3 \mathrm{IU} / \mathrm{L}$, SGPT of 


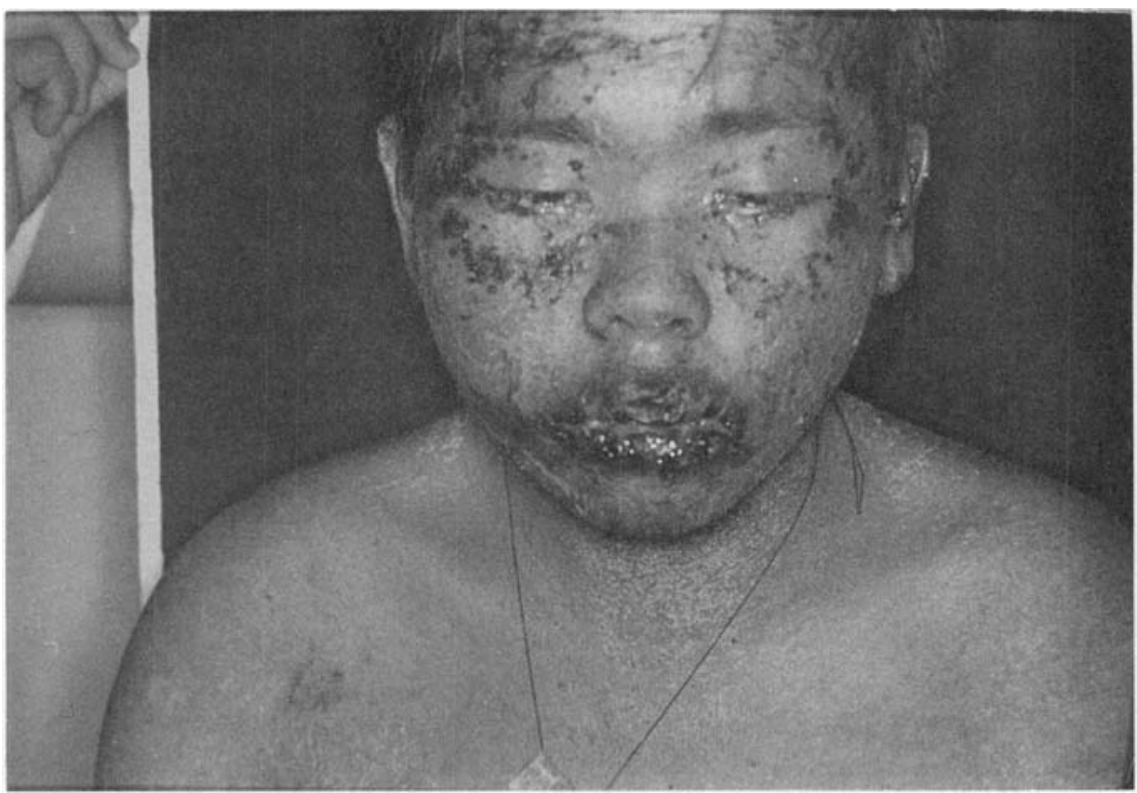

Figure 1. On admission, the patient had icteric sclerae, mucopurulent eye discharge and erosions on the buccal mucosa and lips.

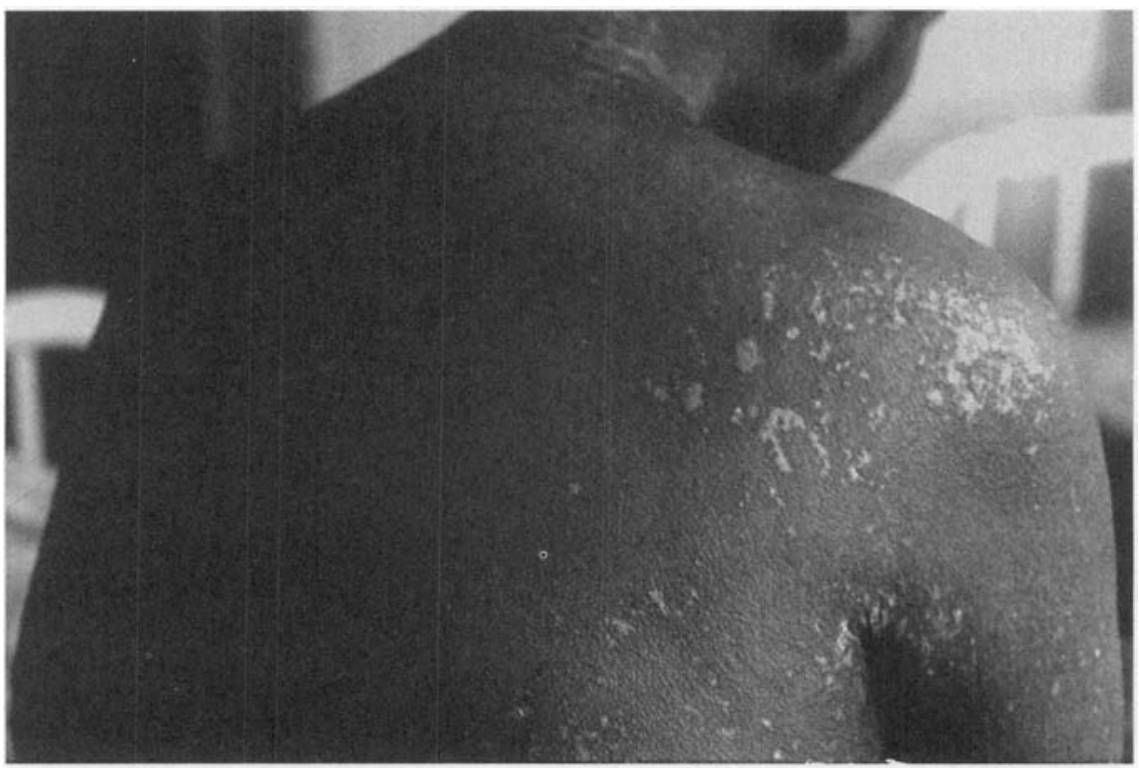

Figure 2. Diff use scaling and erosions at the back of the patient. 


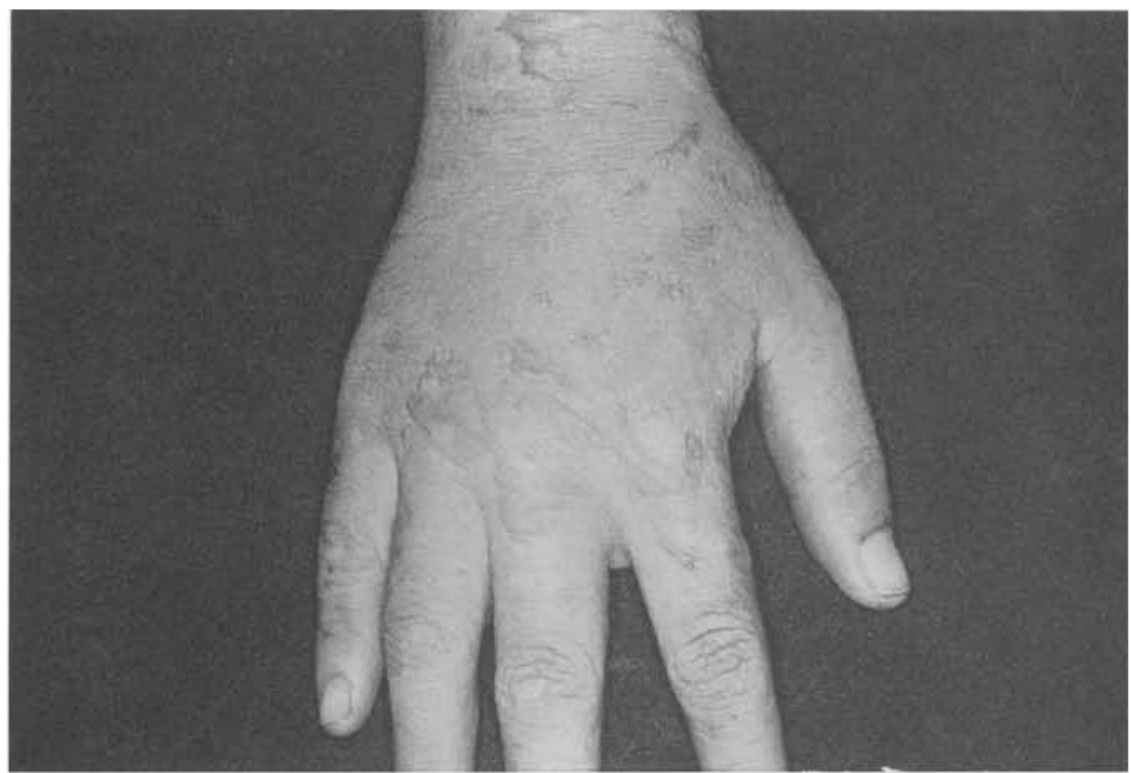

Figure 3. Flaccid bullae on the dorsum of the hand.

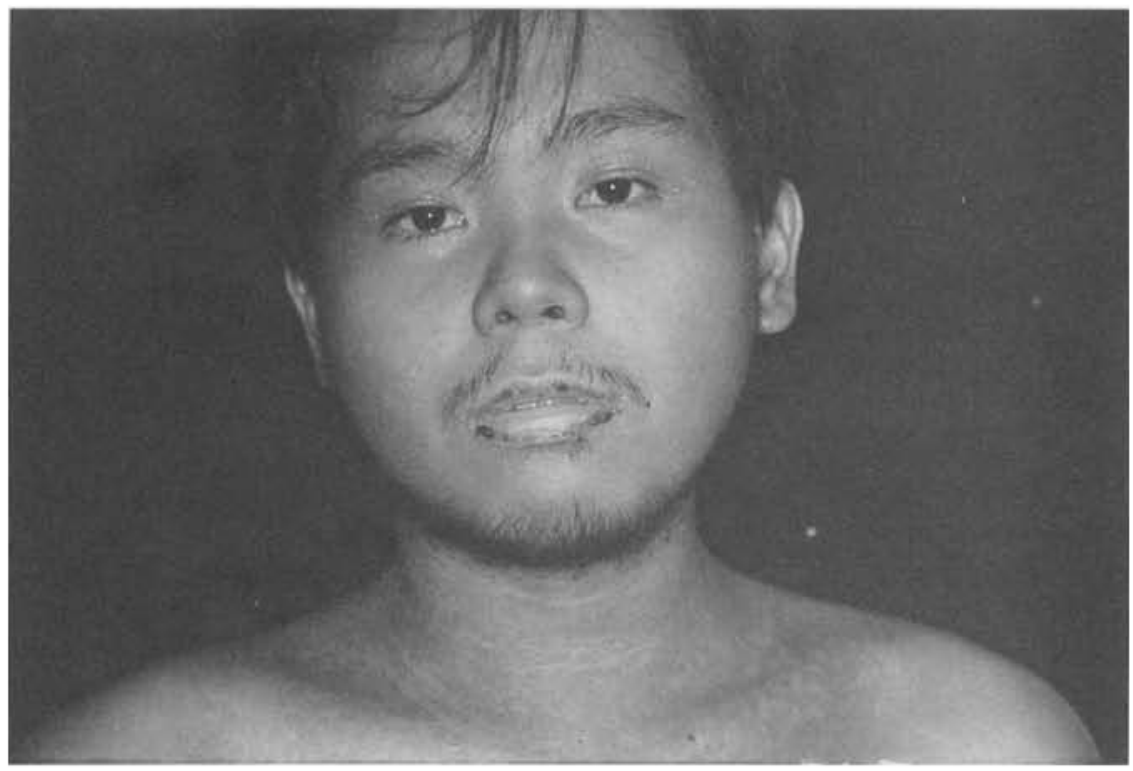

Figure 4. The patient on the 23rd hospital day, prior to discharge, with further clinical improvement. 
$196 \mathrm{IU} / \mathrm{L}$, prolonged prothrombin time of $17 \mathrm{~s}$ (control, 12s). A complete hepatitis profile was nonreactive for all antigens and antibodies. G6PD determination was over $120 \mathrm{~min}$ (normal, 30-60 min). Chest X-ray revealed normal findings. A skin biopsy done on a bulla was consistent with erythema multiforme.

An impression of a hypersensitivity reaction to dapsone was made. All previous medications were discontinued. He was started on intravenous steroid therapy with hydrocortisone at $240 \mathrm{mg} /$ day. This was gradually tapered, then shifted to oral prednisone at $30 \mathrm{mg} /$ day. Chlorpheniramine maleate was also given. Since all the drugs of the WHO-MDT regimen were withdrawn, ofloxacin, a fluoroquinolone with bactericidal properties against Mycobacterium leprae, was given at $400 \mathrm{mg} /$ day. Packed $\mathrm{RBC}$ was transfused for the anaemia and vitamin $\mathrm{K}$ was given for the delayed prothrombin time. He was also given compresses, emollients, betamethasone lotion, tetracycline ointment, and betamethasone and fucidic acid cream.

The patient gradually improved and his skin eruptions cleared with residual hyperpigmentation within 3 weeks from the start of therapy. Upon discharge, haematologic studies and liver function tests were continually monitored and showed normal values within 1.5 months.

Rifampicin and clofazimine were reinstituted with no development of the earlier signs and symptoms observed. He was not challenged with dapsone because of the potential risk of recurrence and severity of his initial illness.

\section{Discussion}

The patient had fever, malaise, anaemia, exfoliative dermatitis and jaundice with gross hepatic dysf unction, all of which developed within 4 weeks after administration of dapsone.

The constellation of symptoms and results of all ancillary procedures favoured the possibility of an adverse drug reaction, most likely due to dapsone. Although rifampicin and clofazimine cannot be readily excluded as the precipitating agents, there have been no published reports attributing a reaction of this type to either drug. Moreover, despite the discontinuation of these two drugs, there was progression of the patient's illness, apparently because of the continuation of dapsone. Likewise there was no recurrence of lesions upon reinstitution of rifampicin and clofazimine.

The earliest report of a hypersensitivity reaction to dapsone was published in 1949. It was thought then that dapsone precipitated glandular fever. The adverse reaction occurred early during the course of treatment and was characterized by fever, lymphadenitis, splenomegaly, jaundice, abnormal liver function tests, mononucleosis and dermatitis, including generalized exfoliation. Diagnosis was confirmed by a positive Paul Bunell test and mononucleosis. Since this reaction was initially associated with high doses of dapsone, the authors suggested a gradual build-up of dapsone to $300 \mathrm{mg}$ daily to avoid this complication. ${ }^{4}$

In $1950^{6}$, Lowe reported three cases of hypersensitivity to the drug and advised early withdrawal of dapsone and the use of antihistamines followed by densensitization. Allday and Barnes in 1951 gave it the name 'dapsone syndrome' and were the first to put forward the view that it was a hypersensitivity reaction. ${ }^{7}$ While the syndrome may appear in an incomplete form, as in this patient in whom lymphadenopathy was not appreciated, Tomecki \& Catalano ${ }^{1}$ have emphasized that dermatitis was always present. 
In 1951 , the frequency of hypersensitivity reaction to dapsone was $2 \%$. Fatal outcomes associated with exf oliation were reported at a dose of $100 \mathrm{mg}$ daily. ${ }^{8}$ From 1956 until 1980, there were only two reports of the dapsone syndrome. Three distinct explanations were offered. Firstly, the reaction had continued to occur but had not been recognized; secondly, the reaction had been recognized but had not been reported; and finally, the reaction in fact virtually disappeared over this period. The first explanation seems unlikely due to its severity while the second is possible, having discussed the problem with mass users of dapsone, although the sudden reappearance of case reports from 1980 onwards emphasized that the hypersensitivity reaction is an unusual occurrence. ${ }^{4}$

One conclusion from these arguments is that the hypersensitivity reaction to dapsone did virtually disappear between 1956 and 1980. One explanation is that the incidence of the reaction is related to dose; in the 1950s, the recommended dose of dapsone was reduced from 300 to $100 \mathrm{mg}$ daily. However, it has been argued that the hypersensitivity reaction to dapsone or to any other drug is not dose dependent. Explanations based on genetics cannot explain sudden changes. Factors related to the manufacture of dapsone have not been investigated. ${ }^{4}$

From 1980 to 1986, there were 27 published case reports suggesting a rise in the frequency of the reaction. ${ }^{4}$ This was further demonstrated in a local study done in Thailand, ${ }^{3}$ which showed an incidence of $3.6 \%$ for the period $1982-88$, a tenfold increase compared with that of $0 \cdot 3 \%$ for the previous period between 1970 and 1982 . The following factors were mentioned as possibly contributing to the observed rise: increased awareness, low dose regimens before 1976, quality of dapsone and combination with other antileprosy drugs. This study showed an apparent increase in hypersensitivity reaction to dapsone after the introduction of MDT and raised the question of a possible drug interaction with rifampicin.

In 1993 in the Philippines, leprosy had a prevalence rate of $2 \cdot 35 / 10,000$ population as compared with a previous $7 \cdot 2 / 10,000$ in 1986.

Javier ${ }^{10}$ in 1970 reported two cases of dapsone hypersensitivity which manifested as erythroderma with accompanying fever and chills followed by generalized coal black hyperpigmentation. To our knowledge, there has been no other documented case of dapsone syndrome as depicted by this case. Although this could be a reflection of a true absence of such an occurrence, the possible inability of the physicians to recognize the syndrome due to lack of awareness cannot be totally discounted.

The cause of the dapsone syndrome remains unknown. It has also been suggested ${ }^{1,7,11}$ that the hypersensitivity reaction is probably not dose related as in the case of toxicity. Even a small dose may be dangerous in a previously sensitized person. Giving the drug by gradually increasing the dose may also not be of help. ${ }^{11}$

With the availability of alternative and effective antileprosy drugs, it is not necessary to desensitize these patients. Moreover, this practice may predispose to dapsone resistance. $^{11}$ The conditions of most patients improve after cessation of dapsone therapy. Indeed, the patient who has been reported in this present paper improved dramatically after cessation of dapsone and institution of systemic steroid therapy.

\section{Conclusion}

The partial or complete expression of the dapsone syndrome as a hypersensitivity 
reaction may be more common than previously thought, and every physician should be familiar with this reaction pattern. Patients on dapsone treatment should be under close supervision and physicians should realize that with any drug-induced reaction, early recognition, prompt withdrawal of the drug and institution of systemic corticosteroids are the important life-saving measures.

\section{Acknowledgments}

I am grateful to the above patient who has been very cooperative. I would like to thank Dr Agnes Espinoza-Thaebtharm and Dr Gertrude P. Chan who were involved in the management of the case. Dr Elizabeth V. Tianco provided comments on the manuscript. Thanks are also due to Dr Antonio Chua for his help in preparing this paper and to Dr Jerry B. Rosario for his encouragement.

\section{References}

1 Tomecki KJ, Catalano CJ. Dapsone hypersensitivity. Arch Derm, 1981; 117: 38-39.

2 Kromann NP, Vilhelmsen K, Stahl D. The dapsone syndrome. Arch Derm, 1982; 118: 531-2.

3 Richardus JH, Smith TC. Increased incidence in leprosy of hypersensitivity reactions to dapsone after introduction of multi-drug therapy. Lepr Rev, 1989; 60: 267-73.

${ }^{4}$ Smith WCS. Are hypersensitivity reactions to dapsone becoming more frequent? Lepr Rev, 1988; 59: 53-8.

5 Jamrozik K. Dapsone syndrome occurring in two brothers. Lepr Rev, 1986; 57: 57-62.

${ }^{6}$ Lowe J. Treatment of leprosy with diamino-diphe nylsulphone by mouth. Lancet, 1950; 1: 145-50.

7 Mohamed KN. Hypersensitivity reaction to dapsone, report from Malaysia. Lepr Rev, 1984; 55: 385-9.

${ }^{8}$ Frey HM, Gershon AA, Borkowsky W, Bullock WE. Fatal reaction to dapsone during treatment of leprosy. Ann Intern Med, 1981; 94: 777-9.

9 Cabanos MG. Epidemiological Indicators of Leprosy as applied to the National Leprosy Control Program. Unpublished paper presented during the Postgraduate course of the Philippine Leprosy Society in Mandaluyong Metro Manila, Philippines, 22-23 Feb. 1994.

10 Javier PR. Hyperpigmentation during dapsone therapy. The Phil Journal of Lepr, 1970; 5: 7-10.

11 Joseph MS. Hypersensitivity to dapsone, four case reports. Lepr Rev, 1985; 56: 315-20. 